\title{
Sperm Protein Associated With the Nucleus on the X Chromosome N1
}

National Cancer Institute

\section{Source}

National Cancer Institute. Sperm Protein Associated With the Nucleus on the X

Chromosome N1. NCI Thesaurus. Code C92987.

Sperm protein associated with the nucleus on the X chromosome N1 (72 aa, $\sim 8 \mathrm{kDa}$ ) is encoded by the human SPANXN1 gene. This protein may be involved in spermatogenesis. 